\title{
Vor dem Numerus clausus steht der Berufswunsch
}

\section{Yvonne Gilli}

Dr. med., Mitglied des FMH-Zentralvorstandes, Departementsverantwortliche Digitalisierung/E-Health

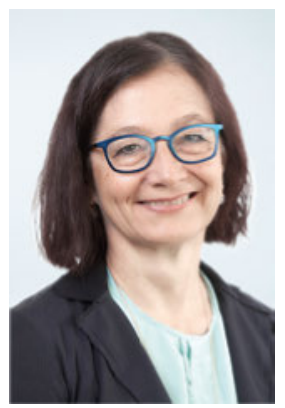

Einmal mehr hat sich das Parlament mit verschiedenen Zulassungskriterien zum Medizinstudium befasst. CVPNationalrätin Ruth Humbel forderte in einer Motion (Mo. 15.3687), dass der Bundesrat eine Alternative zum Numerus clausus prüfe und dabei ein Praktikum als Ersatz oder in Ergänzung zu den Tests der intellektuellen Fähigkeiten einführe. Der Nationalrat hatte die Motion mit grossem Mehr gutgeheissen. Der neu gewählte Bundesrat und Arzt Ignazio Cassis hatte sich der Stimme enthalten. Aber auch er forderte 2015 eine Überprüfung des Numerus clausus mit Verweis auf das israelische Modell (Po. 15.3967). Gemeinsam befürworteten beide ein Selektionsverfahren, das neben den kognitiven Fähigkeiten auch Sozialkompetenz und emotionale Intelligenz für die Zulassung zum Medizinstudium berücksichtigt. Keine Chance hatte die Forderung in der ständerätlichen Kommission für Wissenschaft, Bildung und Kultur, welche den Argumenten der Delegierten der Schweizerischen Hochschulkonferenz sowie der Rektorenkonferenz von swissuniversities folgte. Neben föderalistischen Überlegungen führten diese die Kosten von Praktikumsplätzen sowie die Subjektivität der Beurteilungskriterien als Gegenargumente an.

Eine schöne, allerdings positiv gefärbte Zusammenfassung des Numerus clausus geben Hänsgen et al. vom Zentrum für Testentwicklung und Diagnostik an der Universität Freiburg: Für die Einführung in der Schweiz war das ökonomische Kriterium von besonderem Vorteil. Der Test konnte preisgünstig den deutschen TMS wiederverwerten. Eignungsgespräche und Sozialpraktika wurden als zu teuer verworfen. Mit dem Numerus clausus werden diejenigen bevorzugt zum Medizinstudium zugelassen, welche die besten Aussichten haben, das Studium in einer angemessen kurzen Zeit mit guten Leistungen abzuschliessen. Der Test prüft output-orientiert die Studieneignung, nicht outcome-orientiert die Berufseignung.

Dem Test unterziehen müssen sich allerdings Menschen mit einem Berufswunsch. Es gibt nicht viele Studien, welche nach der Motivation zur Wahl des Arztberufes fragen. Wir wissen, dass Studierende der Medizin vor allem soziale Motive («ich will mit Menschen arbeiten», «Menschen helfen») und wissenschaftliches Interesse («intellektuell anspruchsvolle Tätigkeit») als wichtigste Faktoren für ihre Berufswahl anführen. Gleichzeitig fühlen sie sich in ihrer Berufswahl vergleichsweise sicher. Soziodemografisch fällt auf, dass die Eltern überdurchschnittlich häufig eine akademische Bildung haben und oder selbst im Arztberuf tätig sind (Belastungserleben, Albrecht, 2016). Zu Gunsten des Numerus clausus führen Hänsgen et al. an, dass durch eine intrauniversitäre Selektion mittels Prüfung nach dem ersten Studienjahr wegen der hohen Durchfall-

\section{Der Test (Numerus clausus) prüft output-} orientiert die Studieneignung, nicht outcomeorientiert die Berufseignung.

quote einerseits Lebenszeit nicht erfolgreicher Studierender verschwendet werde, und anderseits der harte Konkurrenzkampf sozial «nicht gerade förderliche Charaktermerkmale» ausbilde. Während letzteres Argument auch gegen den Numerus clausus verwendbar ist, mutet ersteres zynisch an. Immerhin zeigt sich in der Beurteilung durch Pflegefachpersonen ein klares Potenzial für die Verbesserung der interdisziplinaren Zusammenarbeit mit Ärzten und Ärztinnen (Interprofessionelle Zusammenarbeit, Schärli, 2017). Um dieses zu nutzen, braucht es mehr Sozialkompetenz und emotionale Intelligenz vor allem auf der Seite der Ärzteschaft. Übereinstimmung zwischen Politik, universitären Gremien und FMH besteht darin, dass wir auf eine Zulassungsregelung angewiesen sind. Wenig Bewusstsein ist vorhanden für eine integrative Betrachtungsweise, welche den Bogen von der intrinsisch motivationalen Berufswahl über die Gestaltung des Studiums bis zur "guten Ärztin» spannt. Im ständerätlichen Hearing zur Motion Humbel befürwortete die FMH ein zweistufiges Eignungsverfahren, das in Ergänzung zum Numerus clausus Situational Judgment Tests, Multiple Mini-Interviews oder Praktika einführt. 